\title{
Stability of the equation of the $p$-Wright affine functions
}

\author{
JANUSZ BRZDȨK
}

Abstract. We prove some stability results for the equation of the $p$-Wright affine functions.

Mathematics Subject Classification (2010). Primary 39B82; Secondary 39B62.

Keywords. Hyers-Ulam stability, p-Wright convexity, affine function.

\section{Introduction and preliminaries}

Let $0<p<1$ be a fixed real number. We say that a function $f$ mapping a real nonempty interval $I$ into the set of reals $\mathbb{R}$ is $p$-Wright convex provided (see, e.g., $[4,9,16,22])$

$$
f(p x+(1-p) y)+f((1-p) x+p y) \leq f(x)+f(y), \quad x, y \in I .
$$

If $f$ satisfies the functional equation

$$
f(p x+(1-p) y)+f((1-p) x+p y)=f(x)+f(y),
$$

then we say that it is $p$-Wright affine (see [4]).

Note that for $p=1 / 2$ Eq. (1.1) becomes the Jensen's functional equation

$$
f\left(\frac{x+y}{2}\right)=\frac{f(x)+f(y)}{2} .
$$

For $p=1 / 3$ Eq. (1.1) takes the form

$$
f(2 x+y)+f(x+2 y)=f(3 x)+f(3 y),
$$

which has been investigated by Najati and Park [18]; in particular, they proved some results on its stability and applied them in the investigation of the generalized $(\sigma, \tau)$-Jordan derivations on Banach algebras. The cases of more arbitrary $p$ were studied in $[4,5,15]$ (see also $[9,13]$ ).

We prove some results concerning the Hyers-Ulam stability and superstability of (1.1). For more information and numerous references on the stability 
of functional equations we refer to, e.g., [10,14,17,21]; for some examples of various recent outcomes showing new directions in this area of research see, e.g., $[3,7,8,11,12,19,20]$.

The method of the proof of the main result corresponds to some observations in $[6,7,20]$ and the main tool in it is a fixed point result that can be derived from [1, Theorem 1] (see also [2, Theorem 2]). To present it we need the following four hypotheses $\left(\mathbb{R}_{+}\right.$denotes the set of nonnegative reals).

(H1) $X$ is a normed space over a field $\mathbb{F} \in\{\mathbb{R}, \mathbb{C}\}$ ( $\mathbb{C}$ denotes the set of complex numbers) and $Y$ is a Banach space.

(H2) $f_{1}, \ldots, f_{k}: X \rightarrow X$ and $L_{1}, \ldots, L_{k}: X \rightarrow \mathbb{R}_{+}$are given maps.

(H3) $\mathcal{T}: Y^{X} \rightarrow Y^{X}$ is an operator satisfying the inequality

$$
\|(\mathcal{T} \xi)(x)-(\mathcal{T} \mu)(x)\| \leq \sum_{i=1}^{k} L_{i}(x)\left\|\xi\left(f_{i}(x)\right)-\mu\left(f_{i}(x)\right)\right\|
$$

for every $\xi, \mu \in Y^{X}, x \in X$.

(H4) $\Lambda: \mathbb{R}_{+}^{X} \rightarrow \mathbb{R}_{+}{ }^{X}$ is a linear operator defined by

$$
(\Lambda \delta)(x):=\sum_{i=1}^{k} L_{i}(x) \delta\left(f_{i}(x)\right), \quad \delta \in \mathbb{R}_{+}{ }^{X}, x \in X .
$$

Now we are in a position to present the above mentioned fixed point theorem.

Theorem 1.1. Assume that hypotheses (H1)-(H4) are satisfied. Suppose that there are functions $\varepsilon: X \rightarrow \mathbb{R}_{+}$and $\varphi: X \rightarrow Y$ such that

$$
\|(\mathcal{T} \varphi)(x)-\varphi(x)\| \leq \varepsilon(x), \quad x \in X,
$$

and

$$
\varepsilon^{*}(x):=\sum_{n=0}^{\infty}\left(\Lambda^{n} \varepsilon\right)(x)<\infty, \quad x \in X .
$$

Then there exists a unique fixed point $\psi$ of $\mathcal{T}$ with

$$
\|\varphi(x)-\psi(x)\| \leq \varepsilon^{*}(x), \quad x \in X .
$$

Moreover

$$
\psi(x):=\lim _{n \rightarrow \infty}\left(\mathcal{T}^{n} \varphi\right)(x), \quad x \in X
$$




\section{Stability}

The next theorem is the main result in this paper and concerns the stability of Eq. (1.1); it corresponds in particular to some results in [18].

Theorem 2.1. Let (H1) be valid, $p \in \mathbb{F}, A, k \in(0, \infty),|p|^{k}+|1-p|^{k}<1$, and $g: X \rightarrow Y$ satisfy

$$
\begin{aligned}
& \|g(p x+(1-p) y)+g((1-p) x+p y)-g(x)-g(y)\| \\
& \leq A\left(\|x\|^{k}+\|y\|^{k}\right), \quad x, y \in X .
\end{aligned}
$$

Then there exists a unique solution $G: X \rightarrow Y$ of Eq. (1.1) such that

$$
\|g(x)-G(x)\| \leq \frac{A\|x\|^{k}}{1-|p|^{k}-|1-p|^{k}}, \quad x \in X
$$

and $G$ is given by:

$$
G(x):=g(0)+\lim _{n \rightarrow \infty}\left(\mathcal{T}^{n} g_{0}\right)(x), \quad x \in X,
$$

where $g_{0}$ and $\mathcal{T}$ are defined by (2.6) and (2.7).

Moreover, $G$ is the unique solution of Eq. (1.1) such that there exists a constant $M \in(0, \infty)$ with

$$
\|g(x)-G(x)\| \leq M\|x\|^{k}, \quad x \in X .
$$

Proof. Note that $(2.1)$ with $y=0$ gives

$$
\|g(p x)+g((1-p) x)-g(x)-g(0)\| \leq A\|x\|^{k}, \quad x \in X .
$$

Write

$$
g_{0}(x)=g(x)-g(0), \quad x \in X
$$

and

$$
\mathcal{T} \xi(x):=\xi(p x)+\xi((1-p) x), \quad x \in X, \xi \in Y^{X} .
$$

Then (2.5) implies the inequality

$$
\left\|g_{0}(p x)+g_{0}((1-p) x)-g_{0}(x)\right\| \leq A\|x\|^{k}, \quad x \in X,
$$

which means that

$$
\left\|\mathcal{T} g_{0}(x)-g_{0}(x)\right\| \leq A\|x\|^{k}, \quad x \in X .
$$

Further note that (H3) holds with $k=2, f_{1}(x)=p x, f_{2}(x)=(1-p) x, L_{i}(x)=$ 1 for $i=1,2, x \in X$. Define $\Lambda$ as in (H4). Clearly, with $\varepsilon(x):=A\|x\|^{k}$ for $x \in X$, we have 


$$
\begin{aligned}
\varepsilon^{*}(x) & :=\sum_{n=0}^{\infty}\left(\Lambda^{n} \varepsilon\right)(x) \\
& \leq A\|x\|^{k} \sum_{n=0}^{\infty}\left(|p|^{k}+|1-p|^{k}\right)^{n} \\
& =\frac{A\|x\|^{k}}{1-|p|^{k}-|1-p|^{k}}, \quad x \in X .
\end{aligned}
$$

Hence, according to Theorem 1.1, there exists a unique solution $G_{0}: X \rightarrow Y$ of the equation

$$
G_{0}(x)=G_{0}(p x)+G_{0}((1-p) x)
$$

such that

moreover

$$
\left\|g_{0}(x)-G_{0}(x)\right\| \leq \frac{A\|x\|^{k}}{1-|p|^{k}-|1-p|^{k}}, \quad x \in X
$$

$$
G_{0}(x):=\lim _{n \rightarrow \infty}\left(\mathcal{T}^{n} g_{0}\right)(x), \quad x \in X .
$$

Now we show that, for every $x, y \in X, n \in \mathbb{N}_{0}$ (nonnegative integers),

$$
\begin{aligned}
& \left\|\mathcal{T}^{n} g_{0}(p x+(1-p) y)+\mathcal{T}^{n} g_{0}((1-p) x+p y)-\mathcal{T}^{n} g_{0}(x)-\mathcal{T}^{n} g_{0}(y)\right\| \\
& \quad \leq A\left(|p|^{k}+|1-p|^{k}\right)^{n}\left(\|x\|^{k}+\|y\|^{k}\right) .
\end{aligned}
$$

Clearly, the case $n=0$ is just (2.1). Next, fix $m \in \mathbb{N}_{0}$ and assume that (2.10) holds for every $x, y \in X$ with $n=m$. Then

$$
\begin{aligned}
\| \mathcal{T}^{m+1} g_{0}(p x+(1-p) y)+\mathcal{T}^{m+1} g_{0}((1-p) x+p y) & -\mathcal{T}^{m+1} g_{0}(x)-\mathcal{T}^{m+1} g_{0}(y) \| \\
= & \| \mathcal{T}^{m} g_{0}(p(p x+(1-p) y))+\mathcal{T}^{m} g_{0}((1-p)(p x+(1-p) y)) \\
& +\mathcal{T}^{m} g_{0}(p((1-p) x+p y))+\mathcal{T}^{m} g_{0}((1-p)((1-p) x+p y)) \\
& -\mathcal{T}^{m} g_{0}(p x)-\mathcal{T}^{m} g_{0}((1-p) x)-\mathcal{T}^{m} g_{0}(p y)-\mathcal{T}^{m} g_{0}((1-p) y) \| \\
\leq & \left.\left.\| \mathcal{T}^{m} g_{0}(p p x+(1-p) p y)\right)+\mathcal{T}^{m} g_{0}((1-p) p x+p p y)\right) \\
& -\mathcal{T}^{m} g_{0}(p x)-\mathcal{T}^{m} g_{0}(p y) \| \\
& \left.+\| \mathcal{T}^{m} g_{0}(p(1-p) x+(1-p)(1-p) y)\right) \\
& \left.+\mathcal{T}^{m} g_{0}((1-p)(1-p) x+p(1-p) y)\right) \\
& -\mathcal{T}^{m} g_{0}((1-p) x)-\mathcal{T}^{m} g_{0}((1-p) y) \| \\
\leq & A\left(|p|^{k}+|1-p|^{k}\right)^{m}\left(\|p x\|^{k}+\|p y\|^{k}\right) \\
& +\left(|p|^{k}+|1-p|^{k}\right)^{m}\left(\|(1-p) x\|^{k}+\|(1-p) y\|^{k}\right) \\
= & \left(|p|^{k}+|1-p|^{k}\right)^{m+1}\left(\|x\|^{k}+\|y\|^{k}\right), \quad x, y \in X .
\end{aligned}
$$


Thus, by induction we have shown that (2.10) holds for every $x, y \in X$ and $n \in \mathbb{N}_{0}$. Letting $n \rightarrow \infty$ in (2.10), we obtain that

$$
G_{0}(p x+(1-p) y)+G_{0}((1-p) x+p y)=G_{0}(x)+G_{0}(y), \quad x, y \in X .
$$

Write $G(x):=G_{0}(x)+g(0)$ for $x \in X$. Then it is easily seen that

$$
G(p x+(1-p) y)+G((1-p) x+p y)=G(x)+G(y), \quad x, y \in X
$$

and (2.2) holds. It remains to show the statement concerning the uniqueness of $G$.

So suppose that $M_{0} \in(0, \infty)$ and $G_{1}: X \rightarrow Y$ is a solution to (1.1) with

$$
\left\|g(x)-G_{1}(x)\right\| \leq M_{0}\|x\|^{k}, \quad x \in X .
$$

Note that $G(0)=g(0)=G_{1}(0)$,

$$
\begin{aligned}
G_{1}(p x)+G_{1}((1-p) x) & =G_{1}(x)+G_{1}(0), \quad x \in X, \\
G(p x)+G((1-p) x) & =G(x)+G(0), \quad x \in X,
\end{aligned}
$$

and, by (2.2),

$$
\begin{aligned}
\left\|G(x)-G_{1}(x)\right\| & \leq \frac{(M+A)\|x\|^{k}}{1-|p|^{k}-|1-p|^{k}} \\
& =(M+A)\|x\|^{k} \sum_{n=0}^{\infty}\left(|p|^{k}+|1-p|^{k}\right)^{n}, \quad x \in X,
\end{aligned}
$$

where $M:=M_{0}\left(1-|p|^{k}-|1-p|^{k}\right)$.

We show that, for each $j \in \mathbb{N}_{0}$,

$$
\left\|G(x)-G_{1}(x)\right\| \leq(M+A)\|x\|^{k} \sum_{n=j}^{\infty}\left(|p|^{k}+|1-p|^{k}\right)^{n}, \quad x \in X .
$$

The case $j=0$ is exactly (2.13). So fix $l \in \mathbb{N}_{0}$ and assume that (2.14) holds for $j=l$. Then, in view of (2.11) and (2.12),

$$
\begin{aligned}
\left\|G(x)-G_{1}(x)\right\| & =\left\|G(p x)+G((1-p) x)-G_{1}(p x)-G_{1}((1-p) x)\right\| \\
& \leq\left\|G(p x)-G_{1}(p x)\right\|+\left\|G((1-p) x)-G_{1}((1-p) x)\right\| \\
& \leq(M+A)\left(\|p x\|^{k}+\|(1-p) x\|^{k}\right) \sum_{n=l}^{\infty}\left(|p|^{k}+|1-p|^{k}\right)^{n} \\
& \leq(M+A)\|x\|^{k} \sum_{n=l+1}^{\infty}\left(|p|^{k}+|1-p|^{k}\right)^{n}, \quad x \in X .
\end{aligned}
$$

Thus we have shown (2.14). Now, letting $j \rightarrow \infty$ in (2.14) we get $G_{1}=G$. 


\section{A complementary observation on superstability}

The following very simple observation on the superstability of Eq. (1.1) complements Theorem 2.1.

Theorem 3.1. Let (H1) be valid, $p \in \mathbb{F}, A, k \in(0, \infty),|p|^{2 k}+|1-p|^{2 k}<1$, and $g: X \rightarrow Y$ satisfy

$$
\|g(p x+(1-p) y)+g((1-p) x+p y)-g(x)-g(y)\| \leq A\|x\|^{k}\|y\|^{k}
$$

for every $x, y \in X$. Then $g$ is a solution to (1.1).

Proof. Note that (3.1) with $y=0$ gives

$$
g(x)=g(p x)+g((1-p) x)-g(0), \quad x \in X .
$$

We show that, for every $x, y \in X, n \in \mathbb{N}_{0}$,

$$
\begin{aligned}
& \|g(p x+(1-p) y)+g((1-p) x+p y)-g(x)-g(y)\| \\
& \quad \leq A\left(|p|^{2 k}+|1-p|^{2 k}\right)^{n}\|x\|^{k}\|y\|^{k} .
\end{aligned}
$$

Clearly the case $n=0$ is just (3.1). Next, fix $m \in \mathbb{N}_{0}$ and assume that (3.3) holds for every $x, y \in X$ with $n=m$. Then, by (3.2),

$$
\begin{aligned}
\| g(p x & +(1-p) y)+g((1-p) x+p y)-g(x)-g(y) \| \\
= & \| g(p(p x+(1-p) y))+g((1-p)(p x+(1-p) y)) \\
& +g(p((1-p) x+p y))+g((1-p)((1-p) x+p y)) \\
& \quad-g(p x)-g((1-p) x)-g(p y)-g((1-p) y) \| \\
\leq & A\left(|p|^{2 k}+|1-p|^{2 k}\right)^{m}\|p x\|^{k}\|p y\|^{k} \\
& +A\left(|p|^{2 k}+|1-p|^{2 k}\right)^{m}\|(1-p) x\|^{k}\|(1-p) y\|^{k} \\
= & A\left(|p|^{2 k}+|1-p|^{2 k}\right)^{(m+1)}\|x\|^{k}\|y\|^{k}
\end{aligned}
$$

for every $x, y \in X$.

Thus, by induction we have shown that (3.3) holds for every $x, y \in X$ and $n \in \mathbb{N}_{0}$. Letting $n \rightarrow \infty$ in (3.3), we obtain that $g$ is a solution to (1.1), because $|p|^{k}+|1-p|^{k}<1$.

Open Access. This article is distributed under the terms of the Creative Commons Attribution License which permits any use, distribution, and reproduction in any medium, provided the original author(s) and the source are credited.

\section{References}

[1] Brzdęk, J., Chudziak, J., Páles, Zs.: A fixed point approach to stability of functional equations. Nonlinear Anal. 74, 6728-6732 (2011)

[2] Brzdẹk, J., Ciepliński, K.: A fixed point approach to the stability of functional equations in non-Archimedean metric spaces. Nonlinear Anal. 74, 6861-6867 (2011) 
[3] Chudziak, J.: Approximate solutions of the Gołąb-Schinzel functional equation. J. Approx. Theory 136, 21-25 (2005)

[4] Daróczy, Z., Lajkó, K., Lovas, R.L., Maksa, Gy., Páles, Zs.: Functional equations involving means. Acta Math. Hungar. 166, 79-87 (2007)

[5] Daróczy, Z., Maksa, Gy., Páles, Zs.: Functional equations involving means and their Gauss composition. Proc. Am. Math. Soc. 134, 521-530 (2006)

[6] Forti, G.-L.: Comments on the core of the direct method for proving Hyers-Ulam stability of functional equations. J. Math. Anal. Appl. 295, 127-133 (2004)

[7] Forti, G.-L.: Elementary remarks on Ulam-Hyers stability of linear functional equations. J. Math. Anal. Appl. 328, 109-118 (2007)

[8] Forti, G.-L., Sikorska, J.: Variations on the Drygas equation and its stability. Nonlinear Anal. 74, 343-350 (2011)

[9] Gilányi, A., Páles, Zs.: On Dinghas-type derivatives and convex functions of higher order. Real Anal. Exchange 27, 485-493 (2001/2002)

[10] Hyers, D.H., Isac, G., Rassias, Th.M.: Stability of Functional Equations in Several Variables. Birkhäuser, Boston (1998)

[11] Jabłoński, W.: Stability of homogeneity almost everywhere. Acta Math. Hungar. 117, 219-229 (2007)

[12] Jabłoński, W., Reich, L.: Stability of the translation equation in rings of formal power series and partial extensibility of one-parameter groups of truncated formal power series. Sitzungsber. Abt. II 215, 127-137 (2006) Österreichischen Akademie der Wissenschaften (2007)

[13] Jarczyk, W., Sablik, M.: Duplicating the cube and functional equations. Results Math. 26, 324-335 (1994)

[14] Jung, S.-M.: Hyers-Ulam-Rassias Stability of Functional Equations in Nonlinear Analysis. Springer Optimization and Its Applications, vol. 48. Springer, New York (2011)

[15] Lajkó, K.: On a functional equation of Alsina and García-Roig. Publ. Math. Debrecen 52, 507-515 (1998)

[16] Maksa, Gy., Nikodem, K., Páles, Zs.: Results on t-Wright convexity. C. R. Math. Rep. Acad. Sci. Canada 13, 274-278 (1991)

[17] Moszner, Z.: On the stability of functional equations. Aequationes Math. 77, 33-88 (2009)

[18] Najati, A., Park, C.: Stability of homomorphisms and generalized derivations on Banach algebras. J. Inequal. Appl. 2009, 1-12 (2009)

[19] Paneah, B.: A new approach to the stability of linear functional operators. Aequationes Math. 78, 45-61 (2009)

[20] Sikorska, J.: On a direct method for proving the Hyers-Ulam stability of functional equations. J. Math. Anal. Appl. 372, 99-109 (2010)

[21] Ulam, S.M.: Problems in Modern Mathematics. Science Editions. Wiley, New York (1964)

[22] Wright, E.M.: An inequality for convex functions. Am. Math. Monthly 61, 620-622 (1954)

Janusz Brzdȩk

Department of Mathematics

Pedagogical University

Podchorążych 2

30-084 Kraków

Poland

e-mail: jbrzdek@up.krakow.pl

Received: April 23, 2012

Revised: June 25, 2012 